\title{
On the closed form of the array factor for linear arrays
}

\section{Christiansen, Peter Leth}

\section{Published in:}

I E E E Transactions on Antennas and Propagation

Publication date:

1963

\section{Document Version}

Publisher's PDF, also known as Version of record

Link back to DTU Orbit

Citation (APA):

Christiansen, P. L. (1963). On the closed form of the array factor for linear arrays. I E E E Transactions on Antennas and Propagation, 11(2), 198.

\section{General rights}

Copyright and moral rights for the publications made accessible in the public portal are retained by the authors and/or other copyright owners and it is a condition of accessing publications that users recognise and abide by the legal requirements associated with these rights.

- Users may download and print one copy of any publication from the public portal for the purpose of private study or research.

- You may not further distribute the material or use it for any profit-making activity or commercial gain

- You may freely distribute the URL identifying the publication in the public portal

If you believe that this document breaches copyright please contact us providing details, and we will remove access to the work immediately and investigate your claim 
field pattern side-lobe level. In a radar system, the high-resolution and reduction in ambiguity is achieved at the expense of a reduction in power in the main bean when compared with a densely packed array of the same aperture length.

J. V. DiFranco II. L. RuBIX Advanced Studies Dept. Sperry Gyroscope Co. Great Neck, L. I., X. Y.

\section{On the Closed Form of the Array Factor for Linear Arrays*}

Recently Cheng and $\mathrm{Ma}^{1}$ have shown that known relations in $Z$-transform theory developed for sampled-data systems can be used to express the array polynomial for a linear array in a closed form.

The $Z$-transform of a real function $f(t)$ is defined by

$$
Z\{f(t)\}=\sum_{\nu=0}^{\infty} f(\nu T) z^{-\nu}=F(z) .
$$

The array factor for a $n$-element array is

$$
\sum_{\nu=0}^{n-1} \sigma(\nu d) z^{-\nu}=G(z)
$$

where $\sigma(x)$ is the envelope function for the current distribution, and $d$ is the distance between adjacent elements.

When a nonlinear phase envelope function is not included in $\sigma(x), \sigma(x)$ becomes real, and the analogy is established by replacing $t$ by $x, T$ by $d$ and $f(t)$ by $\sigma(x)$. However, the summation is extended to infinity in (1), but not in (2).

Cheng and $\mathrm{Ma}^{1}$ have overcome this difficulty by assuming

$$
\sigma(x)=\sigma(x+(n-1) d)
$$

or

$$
\sigma(x)=-\sigma(x+(n-1) d)
$$

yielding, respectively,

$$
\begin{aligned}
G(z)=\left(1-z^{-(n-1)}\right) & \cdot Z\{\sigma(x)\}+\sigma(0) \Sigma^{-(n-1)}
\end{aligned}
$$

and

$$
\begin{aligned}
G(z)= & \left(1+z^{-(n-1)}\right) \cdot Z\{\sigma(x)\} \\
& -\sigma(0) z^{-(n-1)} .
\end{aligned}
$$

This assumption, however, restricts the envelope functions to periodic ones.

The following alternative procedure removes this limitation. We introduce a unit gate function $\gamma_{n}(x)$ defined by

$\gamma_{n}(x)= \begin{cases}0 & x<0 \text { and } x>(n-1) d \\ 1 & 0 \leq x \leq(n-1) d\end{cases}$

* Received November 21, 1962.

$1 \mathrm{D}$. K. Cheng and $M$. T. Ma, "A new mathematical approach for linear array analysis, IRE Trans. on ANtennas and Propagation, vol. AP-8 pp. 255-259; May, 1960. thereby obtaining the more general formula

$$
G(z)=Z\left\{\sigma(x)_{\gamma n}(x)\right\}
$$

in which the array factor is expressed as a Z-transform.

The usefulness of formula (6) can be illustrated by an example. Consider the envelope function

$$
\sigma(x)=(\omega x)^{p} e^{-a x}
$$

where $p$ is a nonnegative integer, $\omega$ a real number, and $a$ is any real or complex number (yielding envelope functions including trigonometric sine and cosine).

For this envelope function formula (6) yields the array factor

$$
\begin{aligned}
G(\xi) & =Z\left\{(\omega x)^{p} e^{-a x} \gamma_{n}(x)\right\} \\
& =(-\omega)^{p} \frac{\partial^{p}}{\partial d^{p}} \frac{1-e^{-a n d_{z}-n}}{1-e^{-a d_{\xi}^{-1}}}
\end{aligned}
$$

which is closed in $n$, but not in $p$. By means of (8) many useful current distributions can be investigated.

Cheng and $\mathrm{Ma}^{\mathrm{l}}$ have shown that when the current distribution does not involve a nonlinear phase envelope function the numerator and the denoninator in the expression for $|G(z)|^{2}$ always contain the terms $z^{m}$ and $z^{-m}$ in pairs $z^{m}+z^{-m}$.

$$
\text { Lsing }
$$

$$
z=e^{-j \psi}
$$

they introduce

$$
y=z+z^{-1}=2 \cos \psi
$$

and make use of the fact that every pair $z^{m}+z^{-m}$ can be evaluated as a polynomial in $y$.

In the more general case (not considered by Cheng and Ma), ${ }^{1}$ where the current enrelope function $\sigma(x)$ inciudes an amplitude envelope function $r(x)$ and a nonlinear phase envelope function $\phi(x)$,

$$
\sigma(x)=r(x) e^{j \dot{\phi}(x)}=a(x)+j b(x) .
$$

Eq. (2) can be written

$$
\begin{aligned}
G(s) & =\sum_{\nu=0}^{n-1} a(\nu d) s^{-\nu}+j \sum_{\nu=0}^{n-1} b(\nu d) s^{-\nu} \\
& =Z\left\{a(x) \gamma_{n}(x)\right\}+j Z\left\{b(x) \gamma_{n}(x)\right\} .
\end{aligned}
$$

We have found it useful to apply the following relations:

$$
\begin{array}{r}
z^{m}+z^{-m}=P_{m}\left(y^{\prime}\right)=2 T_{m}\left(\frac{y^{\prime}}{2}\right), \\
m=0,1, \cdots \\
j\left(z^{m}-z^{-m}\right)=Q_{m}(y)= \pm 2 U_{m}\left(\frac{y}{2}\right), \\
m=1,2, \ldots .
\end{array}
$$

Plus sign for $\psi \geq 0$;

Minus sign for $\psi \leq 0$ :

where $T_{m}$ and $L_{m}$ are the Chebyshev functions of the first and second kind. ${ }^{2}$

2 T. M. Korn and G. A. Korn, "Mathematical Handbook for Scientists and Engineers," McGrawHill Book Co., Inc., New York, N. Y., p. 723; 1961.
It turns out that in this case the denominator in the expression for $|G(z)|^{2}$ still nay be written as a linear combination of Chebyshev functions of the first kind, while the numerator now also contains linear combinations of Chebyshev functions of the second kind.

It can be shown that if one of the following two conditions,

$$
\begin{aligned}
& r(x)=r((n-1) d-x) \\
& r(x)=-r((n-1) d-x),
\end{aligned}
$$

and the condition

$$
\phi(x)=\phi((n-1) d-x)
$$

are satisfied, $|G(\Xi)|^{2}$ contains no Chebyshev functions of the second kind.

We have investigated several distributions with complex envelope functions as in (11), e.g.,

$$
\begin{aligned}
\sigma(x) & =\left(1+\sin ^{2} \omega x\right) \cdot e^{j 2 \tan ^{-1} \sin \omega x} \\
& =\cos ^{2} \omega x+j \cdot 2 \sin \omega x
\end{aligned}
$$

(no $Q_{m}$-functions are involved in $|G(z)|^{2}$ in this case, if $\omega(n-1) d=1$, because (15a) and (16) are both satisfied) and

$$
\begin{aligned}
\sigma(x) & =\cosh (\omega x+\beta) e^{i \sigma \alpha(\omega x+\beta)} \\
& =1+j \sinh (\omega x+\beta),
\end{aligned}
$$

where $g d$ is the Gudermannian angle.

Peter L. Christiansen Laboratory of Electromagnetic Theory The Technical University of Denmark Copenhagen, Denmark

\section{Designing for Desired Aperture Illuminations in Cassegrain Antennas*}

When designing microwave reflector antennas, one of the major goals is to obtain the desired illumination in the antenna aperture. In Cassegrain antennas, the aperture fieid distribution depends on shape and size of both horn and subreflector, and this apparently makes the design of a desired illumination more involved than in a conventional antenna system using a simple feed horn at the focus. However, a closer look at this problem reveals a possibility to determine the aperture field (and thus also the radiation pattern) without detailed information about horn and subreflector. The only information needed is the type of horn that is to be used, as will be shown below.

The basic geometry of the Cassegrain system is shown in Figs. 1 and 2, as well as the notations used. For simplicity, we assume the horn pattern $g(\phi)$ to be symmetrical, in which case the illumination

\footnotetext{
* Received November 21, 1962.
} 